\title{
Refrigerator cabinet foaming mold accurate design and manufacture based on the vacuum Deformation mechanism
}

\author{
Yuan Hongling ${ }^{1, a}$, Fan Guoquan ${ }^{2, b}$ \\ ${ }^{1}$ Foshan Polytechnic, Foshan, Guangdong, P.R. China 528137 \\ ${ }^{2}$ Xinxiang Polytechnic College, Xinxiang, Henan, P.R. China 453002 \\ ahlyuan0820@163.com, bfgq999@126.com
}

Keywords: Inner shell of the refrigerator, Finite element analysis, Foaming mold, Compensation algorithm

\begin{abstract}
Decreased thickness of refrigerator inner shell makes the surface of refrigerator inner shell have defects such as bulges, wrinkling and deformation after foaming. By using the software of finite element analysis ANSYS, we make a modal analysis on inner shell of the refrigerator. According to the analysis results of deformation of refrigerator inner shell, we modify and compensate the size of the foam mold. Hence, a precise design of refrigerator foaming mold can be achieved.
\end{abstract}

\section{Introduction}

The main parts of the refrigerator comprises of a box body, a door body, electrical system, compressor and refrigeration system and so on. The box body and the door body shell are made by vacuum plastic molding, whose intermediate foam material is made by foam molding. In recent years, since raw material prices continue to rise in national and international markets, reducing production costs and improving product quality are becoming means for a manufacturer to win in the fierce competition. The decrease of the ABS sheet thickness of tank shell and strength reduction will have a great influence on the subsequent processes - foaming of refrigerator. For example, it will make the possibility that refrigerator inner shell have defects such as bulges, wrinkling, deformation increase substantially, which brings difficulty to the precise the design of refrigerator foam mold.

\section{Shortcomings of Conventional Design of Refrigerator Cabinet Foaming Mold}

As one of the main dies in production of refrigerator, because of the big batch its direct influence on the quality of refrigerator body and requirements of assembly process, the design, manufacturing quality and cycle of the refrigerator inner shell foaming die design will directly affect the quality and economic benefit of refrigerator production.

Foaming mold mainly has the following characteristics: it needs universal foaming external fixture (foaming truck), foaming core, a high quality requirement for the model surface, and the structure which influences ejection of mold must adopt drawing core (loose piece) design, and so on [1]. Figure 1 is the drawing of a type of refrigerator cabinet foaming.

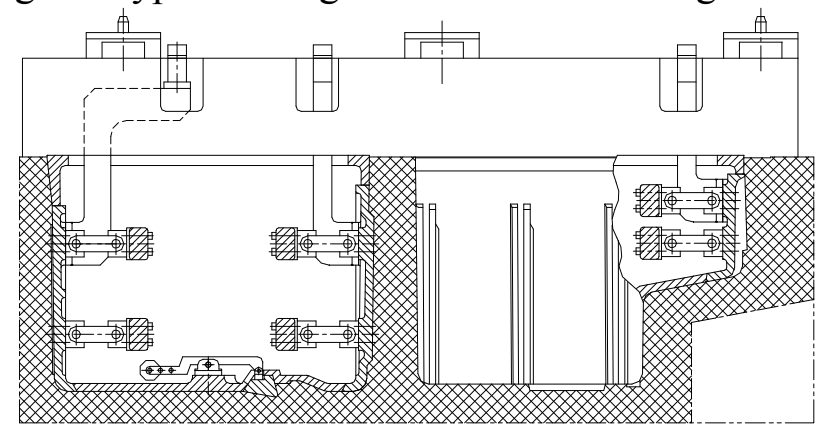

Fig.1 Refrigerator cabinet foaming die 
In the process of new product development, due to insufficient studying in the deep-going way on cooling deformation of the tank shell, we just make a design on the foaming mold according to experience. This relatively simple mold design and debug which makes repeated modifications for blowing mold has been unable to meet the needs of production. At the same time, unreasonable mold design will cause the refrigerator box scrapped. And because of the irreplaceable characteristic of the foaming box, it will cause a rise in the cost of production and directly affect the economic efficiency of enterprises [2].

\section{Finite Element Analysis on Inner Shell of the Refrigerator}

Next, by adopting the theory of thermal elastic-plastic and using the software of finite element analysis ANSYS, taking a certain type of refrigerator box shell as the object of analysis, we make a modal analysis to research the influence of the mold temperature, blowing pressure of plates after heating, and gas pressure of stripping on the box shell size and wall thickness after stretching during the molding process. On that basis, we have a precise design on foaming mold of the box to improve the fit quality of box shell and the box body foam mould, shorten the development cycle, reduce development cost and improve the marketing speed of new products.

Establishment about Solid Model of Inner Shell Structure of the Refrigerator. In order to make use of computer resources more reasonably, we can make some simplifications about structure of subject investigated on condition that the results is consistent with the actual situation. A drawing of a certain type of refrigerator box shell of vacuum forming mold is shown in Figure 2. The simplified model structure of the inner shell of the refrigerator is shown in Figure 3. After the model of inner shell of the refrigerator is established, the constraint relationship of the model is established as well. And the pre-processing module of the ANSYS can be used to build the solid modeling for the inner shell. In other words, in strict accordance with the drawings and production process which are given input of the dimensions, we can get a drawing about the finite element model of inner shell of the refrigerator such as Figure 4.

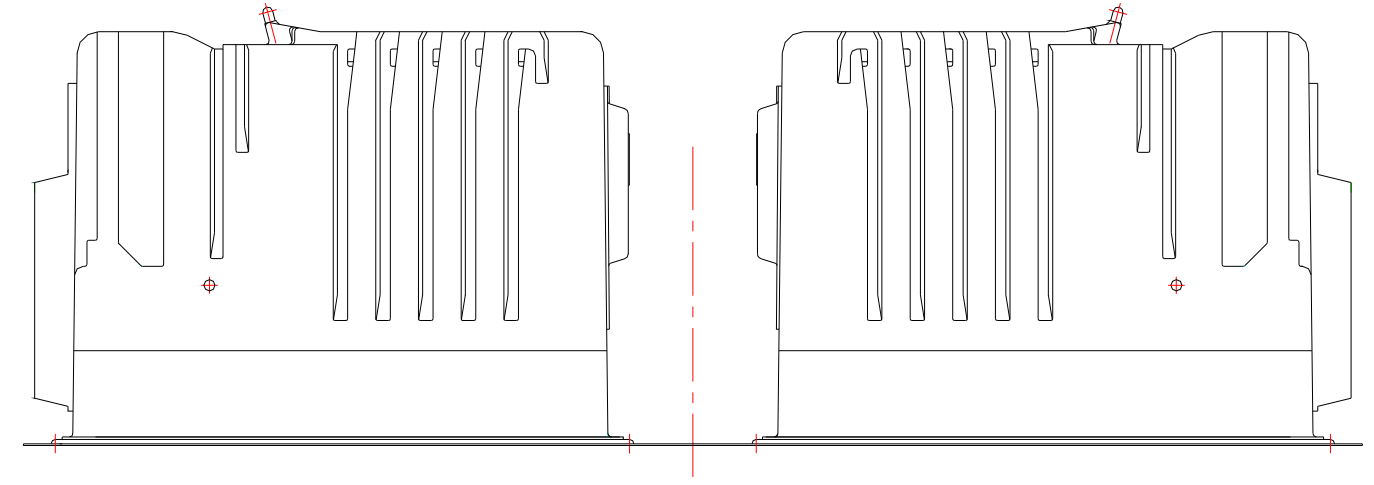

Fig.2 Inner shell of the refrigerator (one die two points)

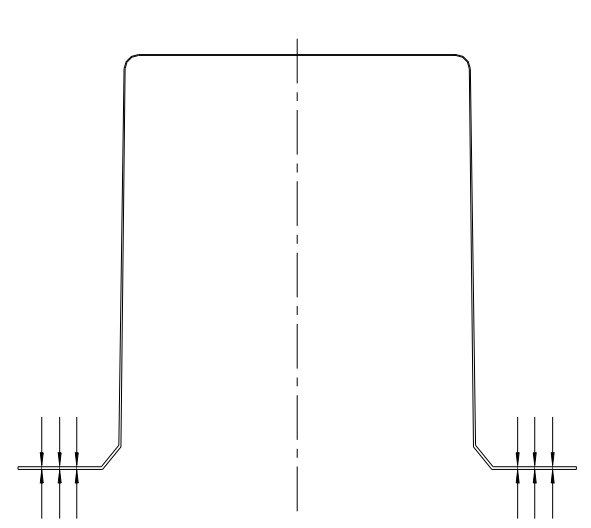

Fig.3 Simplified inner shell box diagram

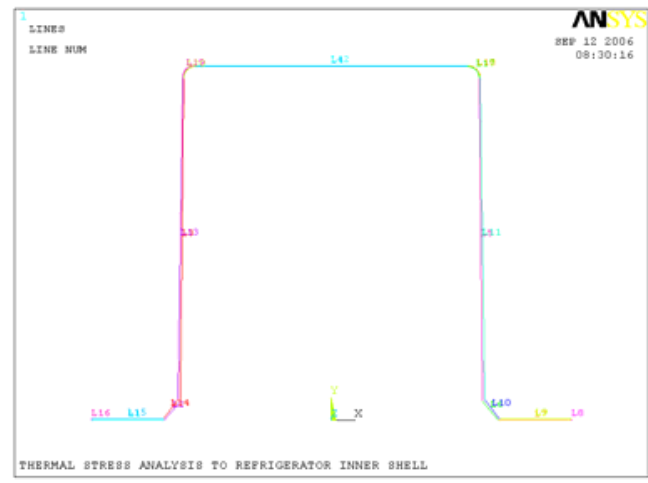

Fig.4 Finite element model of inner shell 
Establishment about Thermodynamic Model of Finite Element of Inner Shell Structure of the Refrigerator. At the completion of solid modeling, we use grid to divide the solid model and establish model of finite element. First of all, we must define the type of element. Because the simplified inner shell of the refrigerator becomes a plane structure, which belongs to the problem of plane strain, we choose SOLID 182element with four nodes to find the solution. Gridding has a great influence on the study of accuracy, and unreasonable partition of grid will even fail to get results. In order to obtain more accurate results, we use a smaller unit to divide the short side and the $\mathrm{R}$ angle of the model, and use a slightly larger cell size to divide the long side [4].The generative model of finite element about inner shell of the refrigerator is shown in figure 5:

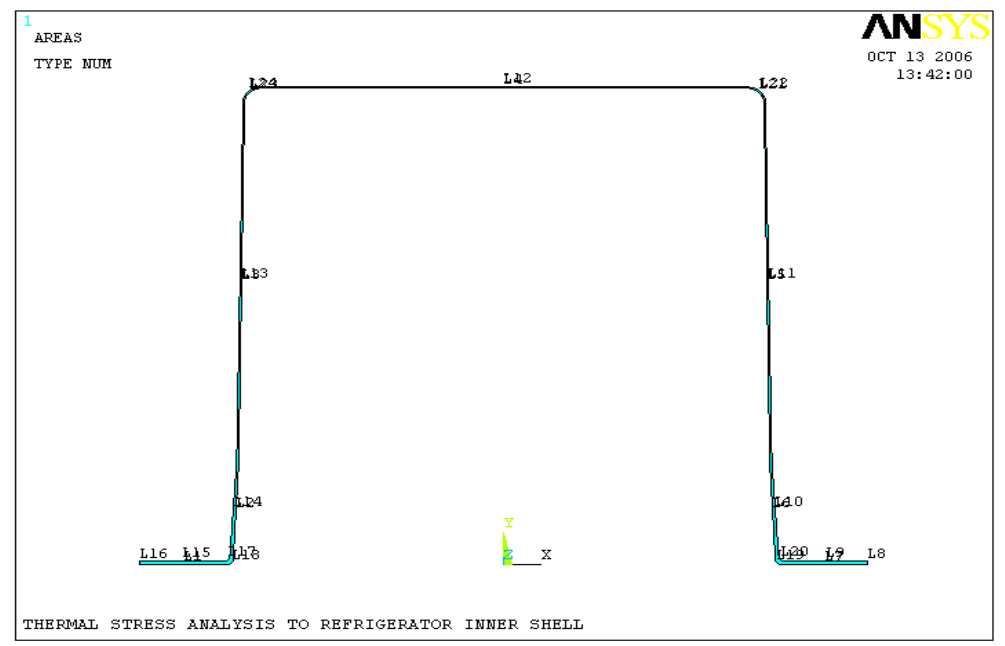

Fig.5 The model of grid generation of inner shell of the refrigerator

The Handling about Load and Constraint. Loads and constraints can be both applied to the model as the determined way. After the load and constraints having been defined, the model will deform and receive force[5].

The technological parameter of inner shell of the refrigerator is of 95 DEG C during forming. Simultaneously, in order to make the heated sheet cool to the desired process temperature rapidly, the interior of mold uses cooling water pipe connected with mold temperature machine. Concurrently, its exterior uses a blower for cooling the cold to cool. Therefore, the starting temperature keeps steady at $95^{\circ} \mathrm{C}$. After molding, by reason of the box shell being very thin, the thickest place is $3 \mathrm{~mm}$ and it can be rapidly cooled to room temperature in air, so we can set room temperature to $20^{\circ} \mathrm{C}$. Consequently, the temperature difference is $75^{\circ} \mathrm{C}$.

Analysis of the Results about Deformation of Inner Shell of the Refrigerator. We get Figure 6 to Figure 9 through the analysis of inner shell of the refrigerator. They are diagrams of combined deformation, deformation map of $\mathrm{X}$ strain, deformation map of $\mathrm{Y}$ strain and diagram in the $\mathrm{Y}$ direction displacement by turns. 


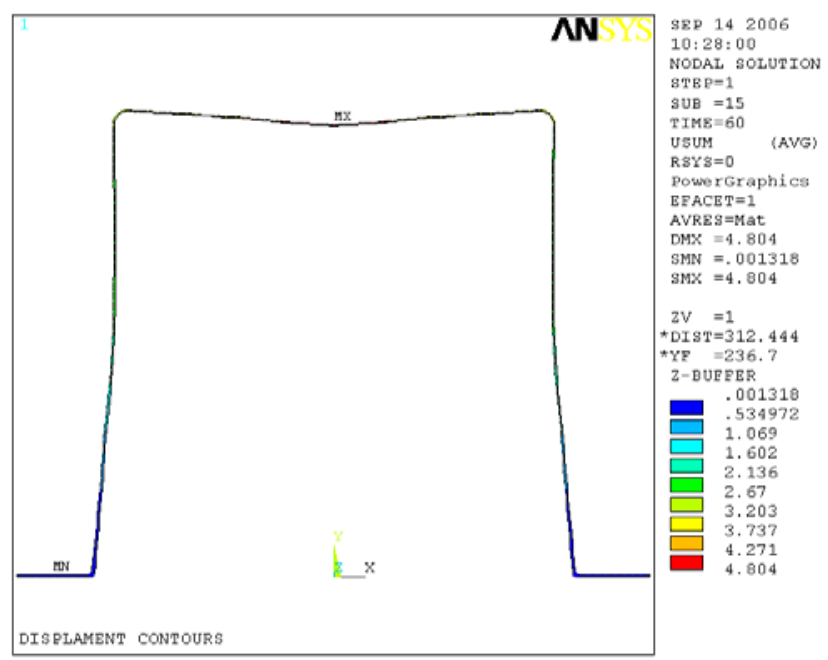

Fig. 6 Diagram of combined deformation about inner shell of the refrigerator

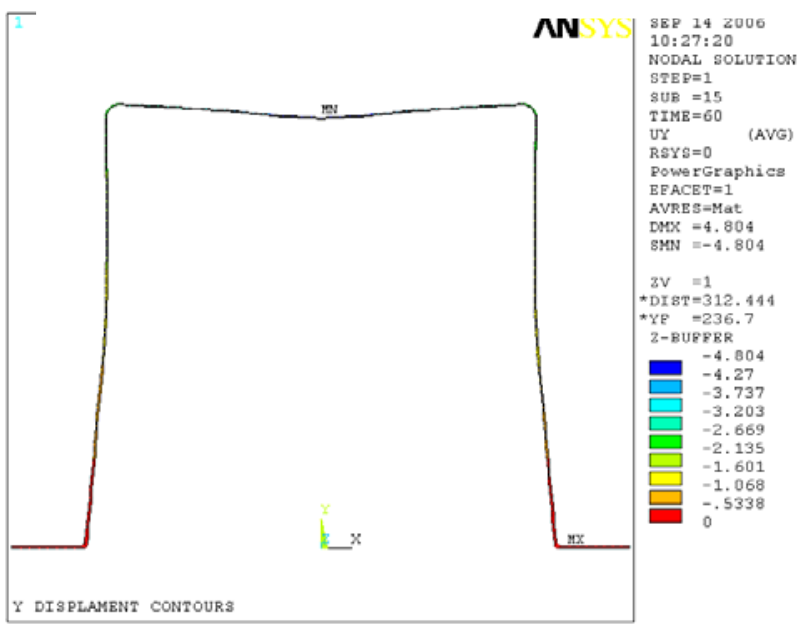

Fig. 7 Deformation map of Y strain about inner shell of the refrigerator

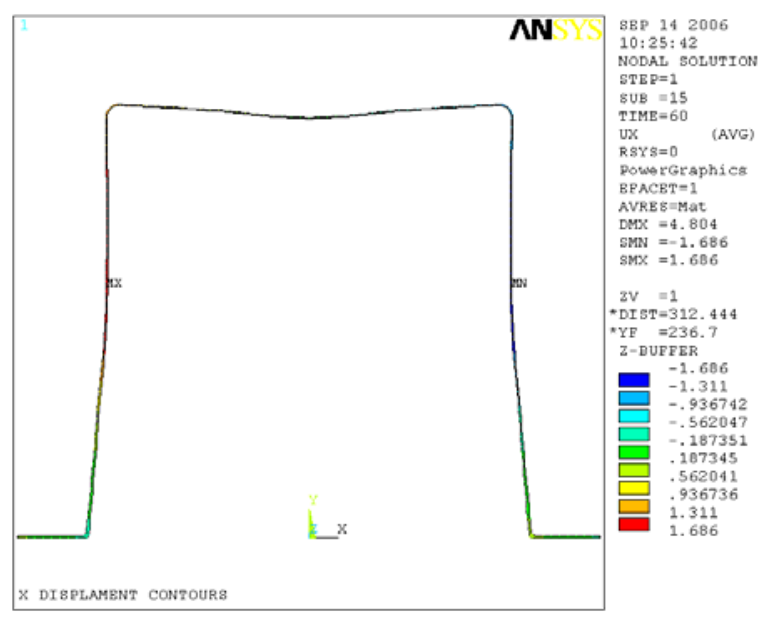

Fig. 8 Deformation map of $\mathrm{X}$ strain about inner shell of the refrigerator

Through analyzing the diagrams above, we get the following tables:

Table 1 Refrigerator inner shell key point deformation chart

Direction Y

\begin{tabular}{|l|l|l|l|l|l|l|l|l|}
\hline & 1,2, & 3 & 4 & 5 & 6 & 7 & 8 & 9 \\
\hline$\Delta \mathrm{Y}$ & 0 & -0.53 & -1.5 & -2.67 & -4.8 & -2.67 & -1.5 & -0.53 \\
\hline $\mathrm{Y}$ & 0 & 100.5 & 245 & 472.4 & 472.4 & 472.4 & 245 & 100.5 \\
\hline$\Delta \mathrm{Y} / \mathrm{Y}$ & $\mathrm{X}$ & $0.53 \%$ & $0.61 \%$ & $0.57 \%$ & $1.02 \%$ & $0.57 \%$ & $0.61 \%$ & $0.53 \%$ \\
\hline
\end{tabular}

Direction X

\begin{tabular}{|l|l|l|l|l|l|l|}
\hline & 1,11 & 2,10 & 3,9 & 4,8 & 5,7 & 6 \\
\hline$\Delta X$ & -0.72 & -0.56 & -0.56 & 1.69 & 1.31 & 0 \\
\hline$X$ & -310 & -231.2 & 100.5 & -245 & -221.2 & 0 \\
\hline$\Delta X / X$ & $0.23 \%$ & $0.24 \%$ & $0.53 \%$ & $0.69 \%$ & $0.59 \%$ & $X$ \\
\hline
\end{tabular}

$\Delta \mathrm{X}$ and $\Delta \mathrm{Y}$ is respectively increment of deformation in $\mathrm{X}$ direction and $\mathrm{Y}$ direction. $\Delta \mathrm{X} / \mathrm{X}$ and $\Delta \mathrm{Y} / \mathrm{Y}$ is respectively percentage of deformation in $\mathrm{X}$ direction and $\mathrm{Y}$ direction. They are the correction factors of the two directions above.

The following conclusions can be drawn from the above table. 
Special point: due to deformation being set up to be zero on the condition, such as 1,2,10,11 in Y may not be considered. Due to point 6 being in axis $Y$ of the coordinate, the deformation of point 6 in $\mathrm{X}$ will not be considered.

As in the middle, the deformation of point 6 in $\mathrm{Y}$ is of maximum distortion.

The deformation of the points such as $1,2,10,11$ in $\mathrm{X}$ are $0.23 \%$ and $0.24 \%$, and they are close to each other. The deformation of remaining points in $\mathrm{X}, \mathrm{Y}$ is ranging from $0.53 \%$ to $0.69 \%$.

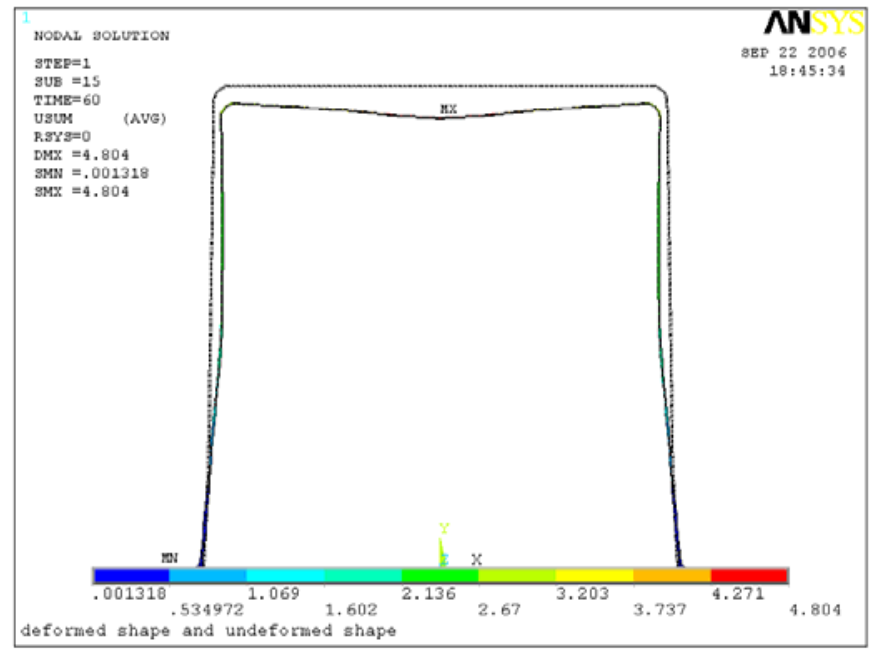

Fig. 9 Displacement diagram of inner shell of the refrigerator

These conclusions can be used as reference for design of foaming mould. In designing of the foaming mold, the larger parts of deformation about inner shell of the refrigerator should be made some corresponding adjustments and modifications so that the shape and size of foaming mold can better adapt to the inner shell of the refrigerator.

\section{Summary}

Through improving the design of foaming mold, mold design, manufacturing, debugging time are shortened from the original 30 to 22days. At the same time, foaming quality is obviously improved. The total of tank body scrapped is greatly reduced.

\section{References}

[1] Z.H. Zhang:China Railway Publishing House,2005 №5, p .3 (In Chinese)

[2] D.H. Zhang, P.L. Yang, Submitted to Journal of Science Technology, 2006 №12, p .65(In Chinese)

[3] D.H. Zhang, P.L. Yang,, Y.Q. Li, Submitted to Journal of Science Technology, 2004 №7, p .76 (In Chinese)

[4] X.C. Wang, M. Shao: The principle and Numerical Method of the Finite Element Method (Tsinghua University Press, Beijing ,2003) (In Chinese)

[5] Y.P. Shi, Y.R. Zhou: Detailed examples of ABAQUS finite element analysis, (Machinery Industry Press , Beijing, 2008) (In Chinese) 